Eliana Aguiar Petri Nahas ${ }^{1}$

Michelle SAKo OMODE $1^{2}$

Luciana Mendes Cangussu ${ }^{3}$

JORGE NAHAS-NETO'

Artigo Original

\title{
Avaliação dos fatores de risco de quedas em mulheres na pós-menopausa inicial
}

\author{
Evaluation of risk factors of falls in early postmenopausal Women
}

\section{Resumo}

OBJETIVO: Foi avaliar a frequência e os fatores de risco de quedas em mulheres na pós-menopausa. MÉTODOS: Estudo clínico, transversal, envolvendo 358 mulheres (idade entre 45 e 65 anos e amenorreia $\geq 12$ meses) com tempo de pós-menopausa $<10$ anos. Os critérios de exclusão foram: doença neurológica ou músculo esquelético, vestibulopatias, hipertensão arterial não controlada, hipotensão postural, déficit visual sem correção, uso de medicamentos (sedativos e hipnóticos). A queda foi definida como mudança de posição inesperada, não intencional, que faz com que o indivíduo permaneça em nível inferior à posição inicial. Foram analisados o histórico de quedas (últimos 24 meses) e as características clínicas, antropométricas (índice de massa corpórea (IMC) e circunferência da cintura (CC) e densidade mineral óssea. Na comparação segundo grupo de mulheres com e sem histórico de queda, foi empregado o Teste do Quiquadrado ou Exato de Fisher e regressão logística com cálculo do odds ratio (OR). RESULTADOS: Entre as mulheres incluídas, 48,0\% (172/358) referiram queda, com fratura em 17,4\% (30/172). A queda ocorreu dentro de casa

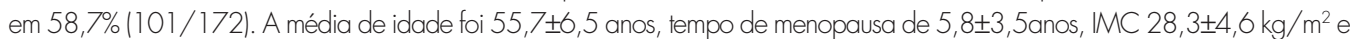
CC $89,0 \pm 11,4 \mathrm{~cm}$. Foi observada maior frequência de tabagismo e diabetes entre as mulheres com histórico de quedas quando comparadas àquelas sem queda, de 25,6 versus $16,1 \%$ e 12,8 versus 5,9\%, respectivamente (p<0,05). Na análise multivariada em função das variáveis clínicas influentes, o risco de queda aumentou com o tabagismo atual (OR 1,93; IC95\% 1,01-3,71). Demais variáveis clínicas e antropométricas não influenciaram no risco de queda. CONCLUSÕES: Em mulheres na pós-menopausa inicial houve expressiva frequência de quedas. $\bigcirc$ tabagismo foi indicador clínico de risco para queda. Com o reconhecimento de fatores determinantes para queda, medidas preventivas são importantes, como a orientação de abolir o tabagismo.

\section{Abstract}

PURPOSE: It was to evaluate the frequency and the risk factors of falls in early postmenopausal women. METHODS: A crosssectional study was conducted on 358 women (age: 45-65 years and amenorrhea $\geq 12$ months) with time since menopause $<10$ years. Exclusion criteria were: neurological or musculoskeletal disorders, vestibulopathies, uncorrected visual deficit, uncontrolled hypertension and postural hypotension, or drug use (sedative and hypnotic agents). A fall was identified as an unexpected unintentional change in position which causes an individual to remain in a lower level in relation to the initial position. The history of self-reported falls during the previous 24 months, and clinical and anthropometric data (body mass index (BMI) and waist circumference (WC) and bone densitometric measures were analyzed. For statistical analysis, $\mathrm{c}^{2}$ trend test and the logistic regression method (odds ratio (OR)) were used for the comparison between groups of women with and without falls. RESULTS: Of the 358 women, 48.0\% (172/358) had a history of falls and 17.4\% (30/172) had fractures. The fall occurred indoors (at home) in $58.7 \%(101 / 172)$. The mean age was $53.7 \pm 6.5$ years, time since menopause $5.8 \pm 3.5$ years, BMl $28.3 \pm 4.6 \mathrm{~kg} / \mathrm{m}^{2}$ and WC $89.0 \pm 11.4 \mathrm{~cm}$. There were differences as the occurrence of smoking and diabetes, with greater frequency among fallers vs. non-fallers, 25.6 versus $16.1 \%$ and 12.8 versus $5.9 \%$, respectively $(p<0.05)$. By evaluating the risk of falls in the presence of influential variables, it was observed that risk increased with current smoking status (OR 1.93; 95\% Cl 1.01-3.711, whereas other clinical and anthropometric variables did not influence this risk. CONCLUSIONS: In early postmenopausal women there was higher frequency of falls. Current smoking was clinical indicators of risk for falls. With the recognition of factors for falling, preventive measures become important, as the orientation of abolishing smoking.

Correspondência

Eliana Aguiar Petri Nahas Departamento de Ginecologia e Obstetrícia - Faculdade de Medicina de Botucatu - Universidade Estadual Paulista - UNESP

Distrito de Rubião Júnior, s/n

CEP: $18650-000$

Botucatu (SP), Brasil

Recebido

$03 / 10 / 2013$
Trabalho realizado no Setor de Climatério e Menopausa do Departamento de Ginecologia e Obstetrícia da Faculdade de Medicina de Botucatu da Universidade Estadual Paulista "Júlio de Mesquita Filho" - UNESP - Botucatu (SP), Brasil.

'Departamento de Ginecologia e Obstetrícia, Faculdade de Medicina de Botucatu,Universidade Estadual Paulista "Júlio de Mesquita Filho" - UNESP - Botucatu (SP), Brasil.

2Faculdade de Medicina de Botucatu, Universidade Estadual Paulista "Júlio de Mesquita Filho" - UNESP - Botucatu (SPI, Brasil.

3Programa de Pós-Graduação em Ginecologia, Obstetrícia e Mastologia, Faculdade de Medicina de Botucatu, Universidade Estadual Paulista "Júlio de Mesquita Filho" - UNESP - Botucatu (SP), Brasil.

Conflito de interesses: não há. 


\section{Introdução}

O aumento na expectativa de vida proporcionou crescimento acentuado da população mais velha, tanto nos países desenvolvidos como naqueles em desenvolvimento. Estima-se que a população de idosos poderá exceder 30 milhões em 20 anos, representando $13 \%$ da população total. Em 2010, foram estimados 19 milhões de idosos, mais da metade composta por mulheres $(55,8 \%)^{1}$. Com o envelhecimento, o equilíbrio postural deteriora em decorrência da diminuição da capacidade funcional do indivíduo. A prevalência de queixas relacionadas ao equilíbrio postural é elevada na população com mais de 65 anos e associada a várias etiologias. Manifesta-se como desequilíbrio, desvios de marcha, instabilidade e quedas, que na população idosa apresentam elevada morbidade e mortalidade ${ }^{2}$. As quedas são responsáveis por $90 \%$ do crescente aumento de fraturas de quadril e são sexta causa de morte entre mulheres acima de 65 anos $^{3,4}$. Reduzir o seu risco é forma de minimizar os custos com assistência à mulher na pós-menopausa, e se torna possível à medida que fatores determinantes das quedas são identificados e intervenções são propostas.

$\mathrm{O}$ hipoestrogenismo parece interferir no equilíbrio postural, explicando o aumento na frequência de quedas e fraturas no período pós-menopausa ${ }^{5-7}$. Embora o sistema muscular e o esquelético sejam estruturalmente interdependentes, em mulheres na pós-menopausa as condições musculares se modificam, alterando a postura, e o centro de gravidade se desloca prejudicando o equilíbrio. A perda do equilíbrio é uma das principais causas de queda, fator precipitante de $90 \%$ das fraturas osteoporóticas de quadril ${ }^{3}$. O risco de uma mulher de 50 anos ou mais apresentar fratura osteoporótica ao longo da vida é de $40 \%$. As fraturas têm sérias consequências em termos de qualidade de vida, morbidade e mortalidade ${ }^{8}$. Uma pesquisa epidemiológica denominada Brazilian Osteoporosis Study (BRAZOS) ${ }^{9}$ avaliou os fatores clínicos de risco para fratura osteoporótica em amostra representativa de 150 diferentes cidades em cinco macrorregiões brasileiras, com total de 2.420 indivíduos (70\% mulheres, $n=1.694$ ). Em mulheres, os principais fatores de risco para fraturas foram: idade avançada, história familiar de fratura de quadril, menopausa precoce, sedentarismo, uso de benzodiazepínicos e queda recorrente. Os autores concluem que intervenções clínicas como estimular atividade física regular e prevenir quedas podem reduzir o risco de fraturas ${ }^{9}$.

Portanto, a prevenção de quedas, para evitar fraturas, deveria ser rotina no atendimento de mulheres na pós-menopausa, pois aproximadamente um terço das mulheres com idade acima dos 60 anos cai pelo menos uma vez ao ano ${ }^{4}$. Nessa população, fatores clínicos relevantes incluem história prévia de queda, fraqueza, sarcopenia, tonturas ou problemas vestibulares, dificuldade de locomoção, artrites ou artroses e déficit visual ${ }^{9}$.
Entretanto, poucos estudos são direcionados para população de mulheres nos primeiros anos de pós-menopausa, necessitando de investigações de possíveis fatores que levam ao evento de quedas. Com base neste contexto, o objetivo deste estudo foi avaliar a frequência e os principais fatores de risco para quedas em mulheres na pós-menopausa inicial.

\section{Métodos}

Trata-se de estudo clínico, analítico e transversal. O grupo populacional foi constituído de pacientes atendidas no Ambulatório de Climatério e Menopausa da Faculdade de Medicina de Botucatu (FMB) - UNESP, de janeiro a dezembro de 2012. O cálculo do tamanho amostral foi embasado no estudo de Barrett-Connor et al. ${ }^{4}$ em que 38,2\% de mulheres na pós-menopausa reportavam pelo menos um episódio de queda. Considerando essa frequência, com nível de significância de 5\% e um erro tipo II de 5\% (poder do teste de $95 \%$ ), foi estimada a necessidade de avaliar, no mínimo, 323 pacientes. Foram incluídas mulheres com: data da última menstruação há pelo menos 12 meses, idade entre 45 e 65 anos, e tempo de pós-menopausa inferior a 10 anos. Os critérios de exclusão foram: doença neurológica com alteração do equilíbrio; doença musculoesquelética com deformidade de membros inferiores; deficiência cognitiva; deficiência visual sem correção; sintomas clínicos de tontura, zumbido, hipoacusia e plentitude auricular (vestibulopatias não controladas); síndrome consuptiva; hipertensão arterial não controlada; hipotensão postural; uso de medicamentos que alterem o equilíbrio (agentes sedativos e hipnóticos); etilismo. Assim, das 559 consultas médicas realizadas em 2012, 221 pacientes foram excluídas por dados incompletos, perimenopausa, idade $<45$ anos e $>65$ anos, ou recusa em participação, de modo que 358 mulheres foram elegíveis para o estudo. Todas as participantes do estudo foram informadas sobre os objetivos da pesquisa, os procedimentos e a confidencialidade dos dados, assinando o Termo de Consentimento Livre e Esclarecido. O projeto foi aprovado pelo Comitê de Ética e Pesquisa da Faculdade de Medicina de Botucatu da UNESP (Of. 456/2011_CEP).

Foram coletadas, por meio de entrevista individual, as seguintes informações: idade, tempo de menopausa, paridade, tabagismo atual, uso ou não de terapia hormonal (TH), acuidade visual, histórico de quedas (últimos 24 meses), de vestibulopatias e de doenças crônicas (hipertensão, diabetes, doença cardiovascular, doenças osteoarticulares), história pessoal de fraturas e atividade física. A queda foi definida como mudança de posição inesperada, não intencional, que faz com que o indivíduo permaneça em nível inferior a posição inicial, não sendo decorrente de paralisia súbita, crise epilética ou força externa ${ }^{10}$. A medida da pressão arterial foi aferida no braço direito com o antebraço apoiado no nível do precórdio, palma da mão 
para cima, com uso de esfigmomanômetro aneroide padrão, com a paciente na posição sentada. Foram consideradas ativas as mulheres que praticaram 30 minutos de exercícios físicos aeróbicos, de intensidade moderada, pelo menos cinco vezes na semana, ou exercícios de força três vezes na semana. A acuidade visual foi avaliada por meio de análise subjetiva com perguntas sobre a interferência da visão na vida diária e uso ou não de óculos.

Foram obtidos os seguintes dados para avaliação antropométrica: peso, altura, índice de massa corpórea (IMC = peso/altura ${ }^{2}$ ) e circunferência da cintura. Para mensuração do peso, foi utilizada balança antropométrica eletrônica microdigital tipo plataforma (Filizola ${ }^{\circledR}$, Brasil), com a paciente descalça e o mínimo de roupa. Para medir a estatura, a paciente permaneceu com os braços ao longo do corpo ereto, mantendo os olhos fixos em plano horizontal paralelo ao chão, medida por haste vertical acoplada à balança. Foram empregados os critérios da Organização Mundial da Saúde (OMS) de 2002 para classificação das pacientes, conforme o IMC: $<24,9 \mathrm{~kg} / \mathrm{m}^{2}$ como normal, de 25 a $29,9 \mathrm{~kg} / \mathrm{m}^{2}$ como sobrepeso e $\geq 30,0 \mathrm{~kg} / \mathrm{m}^{2}$ como obesidade. Para a medida da cintura, foi considerada menor circunferência entre a última costela e a crista ilíaca anterossuperior, sendo a leitura feita no momento da expiração. Foi considerada aumentada acima de $88 \mathrm{~cm}^{11}$.

Para avaliação da densidade mineral óssea (DMO), foram obtidos os laudos de densitometria óssea dos últimos 12 meses realizados pelo Setor de Radiologia da Faculdade de Medicina de Botucatu. A DMO foi medida por meio do exame de absorciometria de raios-X de dupla energia (DEXA) em coluna lombar (L1 a L4) e colo de fêmur, pelo aparelho Hologic QDR-2000 (Waltham, MA, USA). Os valores foram classificados pelo T-score, que corresponde ao valor de DMO média de mulheres jovens normais menos a DMO da paciente, dividido pelo desvio padrão (DP) da média de jovens normais. De acordo com a OMS, o valor de T-score de coluna total e/ou de colo de fêmur $\geq 1,0$ DP é considerado normal; entre -1,0 e -2,4 DP, osteopernia; e $\leq-2,5$ DP, osteoporose ${ }^{12}$.

A partir dos dados, foram construídas as tabelas das variáveis que influenciam o risco de queda, segundo grupo de mulheres na pós-menopausa com e sem histórico de queda. $\mathrm{Na}$ análise descritiva, foram calculados as médias e o desvio padrão para variáveis quantitativas e percentuais para variáveis qualitativas. Para comparação da frequência das características clínicas categorizadas, foi empregado o Teste do Qui-quadrado ou Exato de Fisher de acordo com o parâmetro analisado. Foi realizada análise multivariada por regressão logística binária, considerando-se nível de significância $\mathrm{p}<0,05$ e intervalo de confiança (IC) de 95\%, com cálculo da respectiva odds ratio (OR), considerando o grupo de mulheres com histórico de queda como resposta comparada ao grupo de mulheres sem queda (controle), para se observarem as possíveis associações existentes entre o risco de queda (variável dependente) e as variáveis influentes do risco para queda (variáveis independentes). Foram testadas todas as variáveis estudadas com ajuste do modelo de regressão logística múltipla utilizando procedimento stepwise para as variáveis que apresentaram diferença significativa. Os testes estatísticos foram bilaterais, e o nível de significância adotado foi de 5\%. As análises foram realizadas utilizando-se o Statistical Analyses System (SAS), versão 9.2.

\section{Resultados}

As características descritivas das 358 mulheres na pós-menopausa avaliadas estão apresentadas na Tabela 1 . Observa-se que a média de idade das pacientes incluídas no estudo foi de $55,7 \pm 6,5$ anos com tempo de menopausa de 5,8 $\pm 3,5$ anos. As pacientes foram classificadas como portadoras de sobrepeso (IMC $=28,3 \pm 4,6 \mathrm{~kg} / \mathrm{m}^{2}$ ), com deposição central de gordura. Pela densitometria óssea, observou-se diagnóstico de osteopenia em coluna lombar e colo de fêmur (T-score - 1,4 $\pm 1,3 \mathrm{DP}$ e $-1,1 \pm 1,2 \mathrm{DP}$, respectivamente) (Tabela 1). Observamos que 48,0\% (172/358) referiram queda nos últimos 24 meses, com tempo médio da queda há 12,6 meses. Destas, 17,4\% (30/172) apresentaram fraturas decorrentes da queda. A queda ocorreu dentro de casa em $58,7 \%$ das vezes $(101 / 172)$, na rua em $32,6 \%$ (56/172) e no trabalho em 8,7\% (15/172).

Quanto à associação entre as características clínicas e a frequência de queda, observou-se maior frequência de tabagismo e diabetes entre as mulheres com histórico de quedas quando comparadas àquelas sem esse histórico, de 25,6 versus $16,1 \%$ e 12,8 versus $5,9 \%$, respectivamente $(\mathrm{p}<0,05)$. Em relação a algumas características clínicas, notou-se na população estudada que $9,8 \%$ relatavam idade da menopausa antes de 40 anos (35/358); 84,1\% apresentavam déficit visual (301/358) com correção (uso de óculos); $28,5 \%$ referiram atividade física regular (102/358); $35,7 \%$ eram usuárias de terapia hormonal (128/358);

Tabela 1. Características descritivas, epidemiológicas, clínicas e densitométricas

\begin{tabular}{|c|c|c|c|c|}
\hline Variáveis & Média & DP & Mínimo & Máximo \\
\hline Idade (anos) & 55,7 & 6,5 & 45,0 & 65,0 \\
\hline Idade da menopausa (anos) & 47,4 & 4,6 & 33,0 & 55,0 \\
\hline $\begin{array}{l}\text { Tempo de menopausa } \\
\text { (anos) }\end{array}$ & 5,3 & 3,5 & 1,0 & 9,8 \\
\hline Paridade ( $n^{0}$ de filhos) & 2,5 & 1,8 & 0,0 & 10,0 \\
\hline Peso $(\mathbf{k g})$ & 68,2 & 11,8 & 43,7 & 102,0 \\
\hline Altura (m) & 155,0 & 6,0 & 141,0 & 170,0 \\
\hline IMC (kg/m²) & 28,3 & 4,6 & 18,7 & 41,9 \\
\hline Cintura (cm) & 89,0 & 11,4 & 65,0 & 119,0 \\
\hline DMO coluna total (DP) & $-1,4$ & 1,3 & $-4,5$ & 2,9 \\
\hline DMO colo de fêmur (DP) & $-1,1$ & 1,2 & $-5,3$ & 2,7 \\
\hline
\end{tabular}

DP: desvio padrão; IMC: índice de massa corpórea; DMO: densidade mineral óssea. 
$39,1 \%$ relataram hipertensão arterial $(140 / 358) ; 34,1 \%$ (122/358) apresentaram DMO $\leq-2,0$ DP em coluna lombar e 20,4\% (73/358) em colo de fêmur; não houve diferenças significativas entre os grupos (Tabela 2).

Tabela 2. Associação das características clínicas categorizadas entre as mulheres na pósmenopausa com ( $n=172$ ) e sem histórico de quedas ( $n=186$ )

\begin{tabular}{|c|c|c|c|c|c|}
\hline \multirow{2}{*}{ Características } & \multicolumn{2}{|c|}{ Com queda } & \multicolumn{2}{|c|}{ Sem queda } & \multirow{2}{*}{$\begin{array}{c}\text { Valor } \\
p^{*}\end{array}$} \\
\hline & $\mathrm{n}$ & $\%$ & n & $\%$ & \\
\hline Faixa etária (anos) & & & & & 0,3 \\
\hline$<50$ & 25 & 14,5 & 36 & 19,3 & \\
\hline $50-65$ & 126 & 73,3 & 133 & 71,5 & \\
\hline$\geq 65$ & 21 & 12,2 & 17 & 9,2 & \\
\hline Idade da menopausa (anos) & & & & & 0,2 \\
\hline$\leq \mathbf{4 0}$ & 20 & 11,6 & 15 & 8,1 & \\
\hline$>40$ & 152 & 88,4 & 171 & 91,9 & \\
\hline Tempo de menopausa (anos) & & & & & 0,6 \\
\hline$\leq 5$ & 59 & 34,3 & 73 & 39,2 & \\
\hline $6-10$ & 52 & 30,2 & 50 & 26,9 & \\
\hline$>10$ & 61 & 35,5 & 63 & 33,9 & \\
\hline Tabagismo atual & & & & & 0,03 \\
\hline Sim & 44 & 25,6 & 30 & 16,1 & \\
\hline Não & 128 & 74,4 & 156 & 83,9 & \\
\hline Uso de TH & & & & & 0,4 \\
\hline Sim & 63 & 36,6 & 75 & 40,3 & \\
\hline Não & 109 & 63,4 & 111 & 59,7 & \\
\hline Exercício físico & & & & & 0,1 \\
\hline Sim & 46 & 26,8 & 56 & 30,1 & \\
\hline Não & 126 & 73,2 & 130 & 69,9 & \\
\hline Uso de óculos & & & & & 0,2 \\
\hline Sim & 140 & 81,4 & 161 & 86,6 & \\
\hline Não & 32 & 18,6 & 25 & 13,4 & \\
\hline HAS & & & & & 0,08 \\
\hline Sim & 76 & 44,2 & 64 & 34,4 & \\
\hline Não & 96 & 55,8 & 122 & 65,6 & \\
\hline Diabetes & & & & & 0,03 \\
\hline Sim & 22 & 12,8 & 11 & 5,9 & \\
\hline Não & 150 & 87,2 & 175 & 94,1 & \\
\hline IMC ( $\left(\mathrm{kg} / \mathrm{m}^{2}\right)$ & & & & & 0,4 \\
\hline$\leq 24,9$ & 40 & 23,3 & 53 & 28,5 & \\
\hline $25,0-29,9$ & 67 & 38,9 & 71 & 38,2 & \\
\hline$\geq 30,0$ & 65 & 37,8 & 62 & 33,3 & \\
\hline Cintura (cm) & & & & & 0,3 \\
\hline$<88$ & 76 & 44,2 & 79 & 42,5 & \\
\hline$\geq 88$ & 96 & 55,8 & 106 & 57,5 & \\
\hline DMO coluna lombar (DP) & & & & & 0,4 \\
\hline$>-2,0$ & 109 & 63,4 & 127 & 68,3 & \\
\hline$\leq-2,0$ & 63 & 36,2 & 59 & 31,7 & \\
\hline DMO colo de fêmur (DP) & & & & & 0,3 \\
\hline$>-2,0$ & 133 & 77,3 & 152 & 81,7 & \\
\hline$\leq-2,0$ & 39 & 22,7 & 34 & 18,3 & \\
\hline
\end{tabular}

TH: terapia hormonal; HAS: hipertensão arterial sistêmica; IMC: índice de massa corporal; DMO: densidade mineral óssea; DP: desvio padrão. * Diferença significativa p<0,05 (Teste do Qui-Quadrado).
$\mathrm{Na}$ análise multivariada em função das variáveis clínicas influentes, encontrou-se que o risco de queda aumentou com o tabagismo atual (OR 1,93; IC95\% $1.01-3,71)(\mathrm{p}=0,04)$. Demais variáveis analisadas, como idade (OR 1,01; IC95\% 0,96-1,05), tempo de menopausa (OR 1,05; IC95\% 0,99-1,13), uso de terapia hormonal (OR 0,49; IC95\% 0,14-1,35), visão (OR 1,11; IC95\% 0,96-1,25), antecedente pessoal de diabetes (OR 1,18; IC95\% 0,75-1,87) e hipertensão (OR 1,18; IC95\% $0,75-1,87$ ), IMC (OR 0,88; IC95\% 0,42-1,82), circunferência da cintura (OR 1,18; IC95\% 0,75-1,87), DMO coluna lombar (OR 0,66; IC95\% 0,36-1,22) e colo de fêmur (OR 0,83; IC95\% 0,38-1,81), não influenciaram significativamente no risco de queda $(\mathrm{p}>0,05)$.

\section{Discussão}

Com o aumento da expectativa de vida, espera-se que a frequência de quedas aumente; por isso, a prevenção delas assume especial atenção pelas consequências que acarretam à qualidade de vida de mulheres na pós-menopausa. No presente estudo, demonstrou-se elevada frequência de quedas $(48,0 \%)$ entre as pacientes atendidas em serviço público de saúde. Embora esses resultados não possam ser transferidos para a população geral, esse achado é similar ao observado por outros estudos especificamente com mulheres na pós-menopausa ${ }^{4,13-15}$. Dados de 66.134 mulheres norte-americanas na pós-menopausa participantes do National Osteoporosis Risk Assessment Study (NORA), no qual $58 \%$ das participantes apresentava idade inferior a 65 anos, verificaram que 38,2\% das mulheres reportavam pelo menos um episódio de queda. Os principais fatores de risco foram idade, nível educacional, história pessoal ou familiar de fratura prévia, diabetes e perda de peso ${ }^{4}$. Um estudo brasileiro, que avaliou 4.003 indivíduos com mais de 65 anos, demonstrou frequência de quedas em $34,8 \%$, significativamente maior em mulheres $(40,1 \%)$ que em homens, associando-se diretamente a idade, ao sedentarismo e ao uso crônico de medicamentos ${ }^{16}$.

No resultado de pesquisa sobre quedas em idosos, um bom desempenho físico é mantido até 45-55 anos de idade, dependendo do esforço cobrado; depois dessa faixa etária, um declínio gradual no desempenho é observado ${ }^{17}$. Um aumento nas oscilações posturais foi demonstrado em mulheres com mais de 55 anos, consistente com o aumento de fraturas no antebraço 7 . O presente estudo, ao focar população antes da velhice, média etária de 57,7 anos, não encontrou associação entre a idade e o risco de queda. Esse resultado está em concordância com Rezende et al. ${ }^{14}$, que, avaliando 331 mulheres na pós-menopausa com idade inferior a 65 anos, não demonstraram associação entre a faixa etária e a frequência de queda. Alguns estudos reforçam a idade acima de 65 anos como fator de risco ${ }^{2,10,16}$. 
Todos os componentes do equilíbrio postural - o sensorial, o efetor e o processamento central — são afetados pelo envelhecimento. No sistema sensorial, a acuidade visual e a percepção de profundidade agravam-se com a idade. A visão ajuda a orientar o corpo no espaço ao referenciar os eixos verticais e horizontais dos objetos ao seu redor ${ }^{18}$. Lopez et al. ${ }^{19}$, em estudo australiano, que incluiu 2.340 homens e 3.014 mulheres idosos, demonstraram que o autorrelato de deficiência visual e auditiva associou-se ao aumento no risco de queda e lesões decorrentes delas. Neste estudo, não foi encontrada a acuidade visual como fator de risco, dado que $84,1 \%$ dos indivíduos relataram uso de óculos para correção do déficit visual. Estudos prévios que incluíram a correção da visão no processo multifatorial de avaliação e intervenção sobre o risco de queda obtiveram resultados mistos ${ }^{20,21}$. Não está claro se a visão é componente essencial da intervenção multifatorial. Contudo, se a paciente relatar algum problema, sua visão deve ser avaliada formalmente, e qualquer anormalidade deve ser tratada, em especial a catarata, frequente no idoso ${ }^{22}$.

No presente estudo, apenas $28,5 \%$ das mulheres relataram a prática de atividade física (caminhadas) regular, que não se mostrou como fator de proteção. Contudo, evidências apoiam que exercícios físicos, na forma de treinamento de força, equilíbrio e coordenação, devem ser incluídos como parte de intervenção multifactorial para evitar quedas em idosos ${ }^{23,24}$. A manutenção do equilíbrio implica que a massa e a força muscular estabilizem o corpo evitando a queda. A perda de força e massa muscular está entre as alterações orgânicas mais importantes no processo de envelhecimento, responsável pela diminuição na autonomia e da capacidade funcional do idoso ${ }^{23}$. Contudo, o programa de exercícios deve ser iniciado com cuidado e sob orientação, pois pode aumentar a incidência de quedas de pessoas com mobilidade reduzida e que não estão acostumados à atividade física ${ }^{22}$.

O tabagismo é hoje a principal causa de enfermidades evitáveis e incapacidades prematuras. Dados do Instituto Brasileiro de Geografia e Estatística (IBGE) indicaram que, entre 143 milhões de pessoas acima de 15 anos de idade entrevistadas, 24,6 milhões $(17,2 \%)$ fumavam qualquer produto derivado do tabaco. Entre as mulheres, esse percentual foi de $13,1 \%$ (9,8 milhões $)^{25}$. No presente estudo, encontrou-se que o risco de queda foi associado ao tabagismo atual, observado em $20,7 \%$ das participantes. O equilíbrio de indivíduos fumantes é mais instável que o de não fumantes. A interação entre idade e tabagismo tem efeito significante no aumento da oscilação corporal e no desequilíbrio postural, inferindo-se que os efeitos do tabagismo parecem agravar com o aumento da idade ${ }^{26}$. Contudo, há poucos dados na literatura que avaliam o efeito do tabagismo sobre o risco de queda em mulheres na pós-menopausa. Cangussu et al. ${ }^{15}$, avaliando o equilíbrio postural em 263 mulheres na pós-menopausa com baixa densidade mineral óssea, observaram que o tabagismo foi fator indicador de queda.

O mecanismo de instabilidade do fumante no equilíbrio corporal ainda não está esclarecido. $\mathrm{O}$ tabagismo pode ser responsável por tonturas e dores de cabeça em alguns pacientes com suspeita de envenenamento por monóxido de carbono (CO) presente na fumaça do tabaco ${ }^{27}$. O fumo eleva os valores sanguíneos de carboxihemoglobina a em torno de 10\%; esse nível de CO pode afetar certas funções no sistema nervoso central, como a vigília ${ }^{28}$. Por outro lado, a nicotina deprime os reflexos profundos dos tendões e diminui a atividade gama-eferente, e o controle inadequado do tônus muscular pode afetar proporcionalmente movimentos corretivos para manutenção da postura $^{29}$. Foi demonstrado que fumar um cigarro resulta no aumento da amplitude do tremor posicional; o efeito da nicotina sobre os músculos pode ser responsabilizado pela instabilidade dos fumantes na postura ortostática ${ }^{30}$.

A queda é um evento multifatorial e seus determinantes podem ser atribuídos a fatores extrínsecos, que se relacionam com o ambiente em que o indivíduo se encontra, e a fatores intrínsecos, pertinentes ao estado físico e cognitivo do indivíduo ${ }^{13}$. Entre os fatores extrínsecos, os deslizamentos em pisos escorregadios e a disposição dos móveis na casa são os fatores que mais se relacionam com a queda, as quais tendem a ocorrer com maior frequência no ambiente familiar ${ }^{19,31}$. Na presente pesquisa, observou-se que $58,7 \%$ das mulheres relataram queda dentro de casa. Esse resultado está em conformidade com a literatura. Pesquisas prévias demonstram que o local de maior frequência de quedas é realmente o ambiente domiciliar, entre 53 e $60 \%$ dos episódios ${ }^{13,15,31}$.

Os dados deste estudo apresentam algumas limitações. Por tratar-se de corte transversal, as participantes reportaram dados referentes a quedas retrospectivas, o que pode ter ocasionado algum viés recordatório. Outra limitação é que a maior parte das mulheres é de baixo nível socioeconômico (dois a três salários mínimos) sem acesso à atividade física orientada. A terceira limitação é em relação à população analisada, que foi de pacientes seguidas em ambulatório público específico na assistência à mulher climatérica, em que a TH é rotineiramente indicada para o alívio da sintomatologia climatérica. A proporção de usuárias de $\mathrm{TH}$ $(38,5 \%)$ foi elevada, e a faixa etária predominante foi de mulheres nos primeiros anos de pós-menopausa. Apesar de o tempo de menopausa e o uso de TH não terem se associado ao risco de queda na população avaliada, estudos prévios demonstraram efeitos positivos da terapia hormonal sobre o equilíbrio, com redução do risco de quedas, principalmente se iniciada nos primeiros anos pós-menopausa ${ }^{7,32-34}$.

Em conclusão, o presente estudo revelou número expressivo de quedas em mulheres na pós-menopausa com 
idade inferior a 65 anos. Neste grupo, apenas o tabagismo foi indicador clínico do risco para queda. Assim, na assistência à mulher na pós-menopausa, com o reconhecimento de fatores determinantes para queda, medidas preventivas se tornam importantes, como a orientação para se abolir o tabagismo.

\section{Agradecimentos}

À Fundação de Amparo à Pesquisa do Estado de São Paulo (FAPESP) pelo apoio financeiro da Bolsa de Iniciação Científica, Processo n 2011/19071-0.

\section{Referências}

1. Instituto Brasileiro de Geografia e Estatística [Internet]. Dados do Censo 2010. Rio de Janeiro: IBGE; 2010 [citado 2012 Nov 13]. Disponível em: <http://www.censo2010.ibge.gov.br>

2. Keskin $D$, Borman $P$, Ersöz $M$, Kurtaran $A$, Bodur $H$, Akyüz $M$. The risk factors related to falling in elderly females. Geriatr Nurs. 2008;29(1):58-63.

3. Cummings SR, Melton L. Epidemiology and outcomes of osteoporotic fractures. Lancet. 2002;359(9319):1761-7.

4. Barrett-Connor E, Weiss TW, McHorney CA, Miller PD, Siris ES. Predictors of falls among postmenopausal women: results from the National Osteoporosis Risk Assessment (NORA). Osteoporos Int. 2009;20(5):715-22

5. Ekblad $S$, Bergendhal $A$, Enler $P$, Ledin $T$, Möllen $C$, Hammar $M$. Disturbances in postural balance are common in postmenopausal women with vasomotor symptoms. Climacteric. 2000;3(3):192-8.

6. Bergström I, Ladgren BM, Pyykkö I. Training or EPT in perimenopause on balance and fhushes. Acta Obstet Gynecol Scand. 2007;86(4):467-72.

7. Naessen T, Lindmark B, Lagerström C, Larsen HC, Persson I. Early postmenopausal hormone therapy improves postural balance. Menopause. 2007;14(1):14-9.

8. Management of osteoporosis in postmenopausal women: 2010 position statement of The North American Menopause Society. Menopause. 2010;17(1):25-54.

9. Pinheiro MM, Ciconelli RM, Martini LA, Ferraz MB. Clinical risk factors for osteoporotic fractures in Brazilian women and men: the Brazilian Osteoporosis Study (BRAZOS). Osteoporos Int. 2009;20(3):399-408.

10. Tinetti ME, Speechley M, Ginter SF. Risk factors for falls among elderly persons living in the community. N Engl J Med. 1988;319(26):1701-7.

11. Expert Panel on the Detection, Evaluation, and Treatment of High Blood Pressure in Adults. Executive Summary of the Third Report of the National Cholesterol Education Program (NCEP). Adult Treatment Panel III. JAMA. 2001;285(19):2486-97.

12. Kanis JA. Assessment of fracture risk and its application to screening for postmenopausal osteoporosis: synopsis of a WHO report. WHO Study Group. Osteoporos Int. 1994;4(6):368-81.

13. Silva RB, Costa-Paiva L, Oshima MM, Morais SS, Pinto-Neto AM. [Frequency of falls and association with stabilometric parameters of balance in postmenopausal women with and without osteoporosis]. Rev Bras Ginecol Obstet. 2009;31 (10):496-502. Portuguese.

14. Rezende DAP, Pereira WMP, Schmitt ACB, Pereira ECA, Aldrighi $J M$. Prevalência de quedas em mulheres após menopausa. Rev Bras Crescimento Desenvolv Hum. $2011 ; 21$ (1):146-55.
15. Cangussu LM, Nahas-Neto J, Nahas EAP, Barral ABR, Buttros DA, Uemura $G$. Evaluation of postural balance in postmenopausal women and its relationship with bone mineral density- a cross sectional study. BMC Musculoskelet Disord. 2012;13:2.

16. Siqueira FV, Facchini LA, Piccini RX, Tomasi E, Thumé E, Silveira $\mathrm{DS}$, et al. Prevalência de quedas em idosos e fatores associados. Rev Saúde Pública. 2007;41(5):749-56.

17. El Haber N, Erbas B, Hill KD, Wark JD. Relationship between age and measures of balance, strength and gait: linear and non-linear analyses. Clin Sci (Lond). 2008;1 14(12):719-27.

18. Kärkkäinen $M$, Rikkonen $T$, Kröger $H$, Sirola J, Tuppurainen $M$, Salovaara K, et al. Association between functional capacity tests and fractures: an eight-year prospective population-based cohort study. Osteoporos Int. 2008;19(8):1203-10.

19. Lopez D, McCaul KA, Hankey GJ, Norman PE, Almeida OP, Dobson $\mathrm{A}$, et al. Falls, injuries from falls, health related quality of life and mortality in older adults with vision and hearing impairment - Is there a gender difference? Maturitas. 201 1;69(4):359-64.

20. Gillespie LD, Gillespie WJ, Robertson MC, Lamb SE, Cumming $R G$, Rowe $B H$. Interventions for preventing falls in elderly people. Cochrane Database Syst Rev. 2003;(4):CD000340.

21. Clemson L, Cumming RG, Kendig H, Swann M, Heard R, Taylor K. The effectiveness of a community-based program for reducing the incidence of falls in the elderly: a randomized trial. J Am Geriatr Soc. 2004;52(9): 1487-94.

22. Panel on Prevention of Falls in Older Persons, American Geriatrics Society and British Geriatrics Society. Summary of the Updated American Geriatrics Society/British Geriatrics Society clinical practice guideline for prevention of falls in older persons. J Am Geriatr Soc. 201 1;59(1):148-57.

23. Carter ND, Kannus P, Khan KM. Exercise in the prevention of falls in older people: a systematic literature review examining the rationale and the evidence. Sports Med. 2001;31(6):427-38.

24. Chang JT, Morton SC, Rubenstein LZ, Mojica WA, Maglione M, Suttorp $M$, et al. Interventions for the prevention of falls in older adults: systematic review and meta-analysis of randomised clinical trials. BM. 2004;328(7441):680.

25. Instituto Brasileiro de Geografia e Estatística [Internet]. PNAD 2008 [citado 2012 Fev 10]. Disponível em: <http://www.ibge.gov.br/ home/estatistica/populacao/trabalhoerendimento/pnad2008/ suplementos/tabagismo defautab.shtm>

26. Iki M, Ishizaki $H$, Aalto H, Starck J, Pyykkö I. Smoking habits and postural stability. Am J Otolaryngol. 1994;15(2):124-8.

27. Heckerling PS, Leikin JB, Maturen A, Perkins JT. Predictors of occult carbon monoxide poisoning in patients with headache and dizziness. Ann Intern Med. 1987;107(2):174-6. 
28. Castleden CM, Cole PV. Carboxyhaemoglobin levels of smokers and nonsmokers working in the city of London. Br J Ind Med. 1975;32(2):1 $15-8$.

29. Ginzel KH, Eldred E, Watanabe S, Grover F. Drug-induced depression of gamma efferent activity. I. Peripheral reflexogenic effect of nicotine. Neuropharmacology. 1970;9(2):151-67.

30. Maykoski KA, Rubin MB, Day AC. Effect of cigarette smoking on postural muscle tremor. Nurs Res. 1976;25(1):39-43.

31. Nachreiner NM, Findorff M, Wyman JF, McCarthy TC. Circumstances and consequences of falls in community-dwelling older women. J Womens Health (Larchmt). 2007;16(10): 1437-46.
32. Randell KM, Honkanen RJ, Kroger H, Saarikoski S. Does hormonereplacement therapy prevent fractures in early postmenopausal women? J Bone Miner Res. 2002;17(3):528-33.

33. Barral ABR, Nahas EAP, Nahas-Neto J, Cangussu LM, Buttros DA. Effect of hormone therapy on postural balance in postmenopausal women. Menopause. 2012;19(7):768-75.

34. Bea JW, Zhao Q, Cauley JA, LaCroix AZ, Bassford T, Lewis CE, et al. Effect of hormone therapy on lean body mass, falls, and fractures: 6-year results from the Women's Health Initiative hormone trials. Menopause. $2011 ; 18(1): 44-52$. 